\title{
Branching Pattern and Sequence Analysis of Underivatized Oligosaccharides by Combined MS/MS of Singly and Doubly Charged Molecular Ions in Negative-Ion Electrospray Mass Spectrometry
}

\author{
Wengang Chai \\ MRC Glycosciences Laboratory, Imperial College School of Medicine, Northwick Park Hospital, \\ Harrow, Middlesex, United Kingdom
}

\author{
Alexander M. Lawson \\ MRC Glycosciences Laboratory, Imperial College School of Medicine, Northwick Park Hospital, \\ Harrow, Middlesex, United Kingdom

\section{Vladimir Piskarev} \\ Nesmeyanov Institute of Organoelement Compounds, Russian Academy of Sciences, Moscow, Russia
}

We previously reported that sequence and partial linkage information, including chain and blood-group types, of reducing oligosaccharides can be obtained from negative-ion electrospray CID MS/MS on a quadrupole-orthogonal time-of-flight instrument with high sensitivity and without derivatization (Chai, W.; Piskarev, V.; Lawson, A. M. Anal. Chem. 2001, 73, 651-657). In contrast to oligonucleotides and peptides, oligosaccharides can form branched structures that result in a greater degree of structural complexity. In the present work we apply negative-ion electrospray CID MS/MS to core-branching pattern analysis using nine 3,6branched and variously fucosylated oligosaccharides based on hexasaccharide backbones LNH/LNnH as examples. The important features of the method are the combined use of CID MS/MS of singly and doubly charged molecular ions of underivatized oligosaccharides to deduce the branching pattern and to assign the structural details of each of the 3- and 6-branches. These spectra give complimentary structural information. In the spectra of [M $\mathrm{H}]^{-}$, fragment ions from the 6-linked branch are dominant and those from the 3-linked branch are absent, while fragment ions from both branches occur in the spectra of $[\mathrm{M}-2 \mathrm{H}]^{2-}$. This allows the distinction of fragment ions derived from either the 3- or 6-branches. In addition, a unique $\mathrm{D}_{2 \beta-3}$ ion, arising from double D-type cleavage at the 3-linked glycosidic bond of the branched Gal core residue, provides direct evidence of the branching pattern with sequence and partial linkage information being derived from C- and A-type fragmentations, respectively. (J Am Soc Mass Spectrom 2002, 13, 670-679) (c) 2002 American Society for Mass Spectrometry

$\mathrm{T}$ The potential role of carbohydrates in cellular events has long been hypothesized although evidence for this has only strongly emerged over the last two decades. Awareness of the biological function of oligosaccharide chains in glycoproteins, glycolipids, and proteoglycans has intensified as an increasing number of

Published online April 29, 2002

Address reprint requests to Dr. W. Chai, MRC Glycosciences Laboratory, Imperial College School of Medicine, Northwick Park Hospital, Watford Road, Harrow, Middlesex HA1 3UJ, UK. E-mail: w.chai@ic.ac.uk examples have been reported which reveal that carbohydrate structures participate in various biological events as well as modifying protein functions. One of the early indications of carbohydrates in recognition was binding of the influenza virus to red blood cells via sialic acid [1], and later by work on the chemical basis of the antigenicity of polysaccharides and of the well-known $\mathrm{ABO}(\mathrm{H})$ bloodgroup system [2,3] in which specificity is determined by oligosaccharides. Carbohydrates are well placed to act in cellular recognition as many cells are surrounded by an oligosaccharide layer from cell associated glyco- 
conjugates that often overshadows protein and lipid components on the cell surface.

Specific oligosaccharide sequences, such as the type 1 (Gal $\beta 1-3 \mathrm{GlcNAc}$ )/type 2 (Gal $\beta 1-4 \mathrm{GlcNAc}$ ) chains and the blood group-related antigens bearing the $\mathrm{H}$ (Fuc $\alpha 1-$ 2 Gal $\beta 1-3 / 4$ GlcNAc), Lewis ${ }^{\mathrm{a}}$ [Le ${ }^{\mathrm{a}}$, Gal $\beta 1-3$ (Fuc $\left.\alpha 1-4\right)$ GlcNAc], and Lewis ${ }^{x}$ [Le ${ }^{x}$, Gal $\beta 1-4$ (Fuc $\left.\alpha 1-3\right)$ GlcNAc] determinants, occur naturally on the carbohydrate chains of glycoproteins and glycolipids and comprise recognition motifs for cell-cell and cell-matrix interactions [4, 5]. In lymphocyte adhesion, for example, $\mathrm{Le}^{\mathrm{a}} / \mathrm{Le}^{\mathrm{x}}$ and related sialylated and sulfated oligosaccharide sequences are important ligands [6, 7]. Methods for detailed characterization of these recognition motifs are important in modern structural cell biology to derive structure/function relationships, particularly in the postgenomic era, in order to understand posttranslational glycosylation and its function.

With small amounts of material, no single analytical technique is capable of the complete characterization of oligosaccharide structure. As a consequence, structure elucidation is usually performed by using several different techniques, of which mass spectrometry and NMR are two of the most powerful. Mass spectrometry has been a primary technique in carbohydrate structural analysis [8] for more than three decades, with the information available depending strongly on instrumentation and ionization methods. Electrospray (ES) ionization and matrix-assisted laser desorption/ionization (MALDI) have permitted mass spectrometry to be used not only to measure molecular mass but also to determine sequence, branching pattern, and linkage of oligosaccharides [9-24].

Neutral underivatized oligosaccharides have been analyzed directly by MALDI $[23,24]$ while derivatization (e.g., permethylation [10-13] or reducing-terminal derivatization [14-19]) or metal ion complexation/cationization [20] is generally considered necessary to generate useful structural information and to achieve high sensitivity detection in ES-MS [21]. However, we [25] and others [26] were able to demonstrate high sensitivity detection of underivatized oligosaccharides in both negative- and positive-ion ES-MS. Negative-ion detection is our preferred method and is advantageous for oligosaccharide analysis because of the low chemical background noise [27] and the low level of cation adduct formation [28].

Previously, we demonstrated distinction of chain type and also blood-group type (such as $\mathrm{Le}^{\mathrm{a} / \mathrm{x}}$ and $\mathrm{Le}^{\mathrm{b} / \mathrm{y}}$ ) by ES CID MS/MS in the negative-ion mode with high sensitivity without derivatization [25]. Several characteristic fragmentations are useful to derive detailed structural information. The double glycosidic D-type cleavage is unique to 3-linked GlcNAc and Glc, resulting in fragment ions indicating type 1 chain or blood group types. Double carbon-carbon bond ${ }^{0,2} \mathrm{~A}-$ cleavages (see Results and Discussion for nomenclature of fragmentation) occur with 4-linked GlcNAc and Glc. For example, a D-fragment at $\mathrm{m} / \mathrm{z} 202$ indicates a type 1 chain while an ${ }^{0,2} \mathrm{~A}$-ion doublet at $\mathrm{m} / \mathrm{z} 281 / 263$ indicates a type 2 chain. D-ions at $\mathrm{m} / \mathrm{z} 348$ or $\mathrm{m} / \mathrm{z} 364$, together with a C-ion at $m / z 528$, are characteristic of either a $\mathrm{Le}^{\mathrm{a}}$ or a $\mathrm{Le}^{\mathrm{x}}$ determinants, respectively.

In contrast to oligonucleotides and peptides, oligosaccharides can form branched structures and hence, a relatively simple set of monosaccharides can form a huge number of complex structures. A greater degree of structural complexity produced by branching is the norm for naturally occurring carbohydrates, and frequently a branched sequence carrying two or more recognition motifs are more potent $[6,7]$. Although the branching pattern can be identified in the mass spectra of some derivatized oligosaccharides [27], detailed assignment of a specific chain branch and its linkage to the core sugar residue often needs more specialized methods. 2D-NMR spectroscopy is the technique of choice but sensitivity is limited. Microscale chemical oxidative cleavage of the terminal branching monosaccharide residue in reduced form combined with MS analysis has been used successfully for mucin-type $O$-linked glycoprotein oligosaccharides [29, 30] and mannitol-terminating oligosaccharides released from brain glycoproteins [31].

In the present report, we extend the negative-ion electrospray CID MS/MS studies to core-branching pattern analysis using nine 3,6-branched oligosaccharides isolated from human milk based on hexasaccharide backbones LNH/LNnH (Table 1). Variously fucosylated structures are used to demonstrate the application of the method for assigning structural details, including sequence, partial linkage, and chain and blood-group typing, of each of the 3- and 6-branches.

\section{Experimental}

\section{Materials}

Oligosaccharides were isolated from human milk obtained from a healthy 25-year-old woman, blood group $\mathrm{B}$ secretor, $\mathrm{Le}^{\mathrm{b}}$ positive, giving negative reaction in hepatitis B and HIV tests. Fat was removed by centrifugation at $4{ }^{\circ} \mathrm{C}(5000 \mathrm{~g}, 30 \mathrm{~min})$ and proteins by precipitation with cold ethanol. Oligosaccharides were separated from lactose on a Sephadex G-25 column $(5 \times$ $90 \mathrm{~cm}$ Amersham Pharmacia Biotech AB, Uppsala, Sweden), and then neutral from acidic oligosaccharides on a Dowex 1X2 (100-200 mesh, acetate form Bio-Rad Laboratories, Richmond, CA) column. Gel filtration chromatography was carried out on Fractogel HW40(S) and the partially resolved hexa- (F6), hepta- (F7), octa- (F8), and nonasaccharide (F9) fractions were further fractionated by normal phase HPLC on an amino column. Each subfraction was purified by reversedphase HPLC. Lacto- $N$-hexaose (LNH) and lacto-N-neohexaose ( $\mathrm{LNnH}$ ) were obtained from fraction $\mathrm{F} 6$, monofucosyllacto- $N$-hexaose III (MFLNH III) from fraction F7, difucosyllacto- $N$-hexaoses [a], [b], and [c] (DFLNH $[\mathrm{a}],[\mathrm{b}]$, and $[\mathrm{c}]$, respectively) and difucosyllacto-N-neo- 
Table 1. Structures of branched oligosaccharides used for negative-ion ES-CID-MS/MS

\begin{tabular}{|c|c|c|c|}
\hline Oligosaccharides & Sequences & Chain types $^{a}$ & Blood group determinants \\
\hline $\begin{array}{l}\text { Lacto-N-hexose } \\
\text { LNH }\end{array}$ & $\begin{array}{l}\text { Gal } \beta 1-4 G \mid c N A c \beta 1 \\
\text { Gal } \beta 1-3 G I c N A c \beta 1\end{array}$ & $2 ; 1$ & $-;-$ \\
\hline $\begin{array}{l}\text { Lacto-N-neohexaose } \\
\text { LNnH }\end{array}$ & $\begin{array}{l}\text { Gal } \beta 1-4 G \mid c N A c \beta 1 \\
\text { Gal } \beta 1-4 G \mid c N A c \beta 1\end{array}$ & $2 ; 2$ & $-;-$ \\
\hline $\begin{array}{l}\text { Monofucosyllacto-N-hexaose III } \\
\text { MFLNH III }\end{array}$ & $\begin{array}{c}\text { Fuc } \alpha 1 \\
3 \\
\text { Gal } \beta 1-4 \mathrm{GlcNAc} \beta 1 \\
\text { Gal } \beta 1-3 \mathrm{GlcNAc} \beta 1\end{array}$ & $2 ; 1$ & $\mathrm{Le}^{\mathrm{x}} ;-$ \\
\hline $\begin{array}{l}\text { Monofucosyllacto-N-neohexaose [a] } \\
\text { MFLNnH [a] }\end{array}$ & $\begin{array}{c}\text { Fuc } \alpha 1 \\
3 \\
\text { Gal } \beta 1-4 \mathrm{GlcNAc} \beta 1 \\
\text { Gal } \beta 1-4 \mathrm{GlcNAc} \beta 1^{\prime}\end{array}$ & $2 ; 2$ & $\mathrm{Le}^{\mathrm{x}} ;-$ \\
\hline $\begin{array}{l}\text { Difucosyllacto-N-hexaose [b] } \\
\text { DFLNH [b] }\end{array}$ & $\begin{array}{c}\text { Fuc } \alpha 1 \\
3 \\
\text { Gal } \beta 1-4 G I c N A c \beta 1 \\
\text { Gal } \beta 1-3 G I c N A c \beta 1 \\
4 \\
\text { Fuc } \alpha 1\end{array}$ & $2 ; 1$ & $L e^{x} ; \operatorname{Le}^{a}$ \\
\hline $\begin{array}{l}\text { Difucosyllacto-N-neohexaose } \\
\text { DFLNnH }\end{array}$ & $\begin{array}{c}\text { Fuc } \alpha 1 \\
3 \\
\text { Gal } \beta 1-4 \mathrm{GlcNAc} \beta 1 \\
\text { Gal } \beta 1-4 \mathrm{GIcNAc} \beta 1 \\
3 \\
\text { Fuc } \alpha 1\end{array}$ & $2 ; 2$ & $\operatorname{Le}^{x} ; \operatorname{Le}^{x}$ \\
\hline $\begin{array}{l}\text { Difucosyllacto-N-hexaose [a] } \\
\text { DFLNH [a] }\end{array}$ & 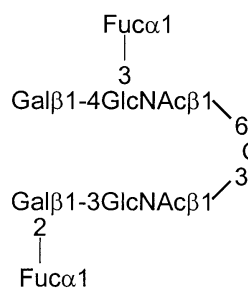 & $2 ; 1$ & $\mathrm{Le}^{\mathrm{x}} ; \mathrm{H}$ \\
\hline $\begin{array}{l}\text { Difucosyllacto-N-hexaose [c] } \\
\text { DFLNH [c] }\end{array}$ & $\begin{array}{c}\text { Gal } \beta 1-4 G \mid c N A c \beta 1 \\
\text { Gal } \beta 1-3 G \mid c N A c \beta 1 \\
\text { Fuc } \alpha 1 \\
\text { Fuc } \alpha 1 \\
\text { Fuc } \alpha 1\end{array}$ & $2 ; 1$ & $-; L^{b}$ \\
\hline $\begin{array}{l}\text { Trifucosyllacto-N-hexaose } \\
\text { TFLNH }\end{array}$ & 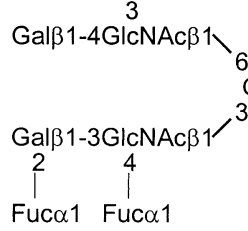 & $2 ; 1$ & $L e^{x} ; L^{b}$ \\
\hline
\end{tabular}

${ }^{a}$ Figures indicate chain types on 6- and 3-branch, respectively.

bBlood-group determinants on 6- and 3-branch, respectively. 
hexaose (DFLNnH) from fraction F8, and trifucosyllacto$N$-hexaose (TFLNH) from fraction F9. Monofucosyllacto$N$-neohexaose [a] (MFLNnH [a]) was prepared from monofucosyl- monosialyllacto- $N$-neohexaose after removal of $N$-acetylneuraminic acid residue by neuraminidase from Clostridium perfringens. Structure and purity of the oligosaccharides was determined by ${ }^{1} \mathrm{H}-\mathrm{NMR}$ (500 MHz) spectroscopy (Brucker WM-500) and detailed characterization will be published elsewhere.

\section{Electrospray Mass Spectrometry}

Negative-ion ES-MS and collision induced dissociation (CID) MS/MS was carried out on a Micromass (Manchester, UK) Q-Tof mass spectrometer. Nitrogen was used as desolvation and nebulizer gas at a flow rate of $250 \mathrm{~L} / \mathrm{h}$ and $15 \mathrm{~L} / \mathrm{h}$, respectively. Source temperature was $80^{\circ} \mathrm{C}$ and the desolvation temperature $150{ }^{\circ} \mathrm{C}$. Typically, a cone voltage of $70 \mathrm{~V}$ was used for CID MS/MS of singly charged ions $[\mathrm{M}-\mathrm{H}]^{-}$and 30-40 V for doubly charged ions $[\mathrm{M}-2 \mathrm{H}]^{2-}$. The energy of 70 $\mathrm{V}$ was sufficient to produce fragment ions and hence, was also used to obtain a product-ion spectrum in quasi $\mathrm{MS}^{3}$ mode of the double glycosidic cleavage ion $\mathrm{D}_{2 \beta-3}$ as the precursor. The capillary voltage was maintained at $3 \mathrm{kV}$. Product-ion spectra were obtained from CID using argon as the collision gas at a pressure of $1.7 \mathrm{bar}$. The collision energy was adjusted between 13-29 V for optimal fragmentation and, typically, 23-29 V was used for CID of $[\mathrm{M}-\mathrm{H}]^{-}, 18-20 \mathrm{~V}$ for $[\mathrm{M}-\mathrm{H}]^{2-}$, and 13-15 $\mathrm{V}$ for CID of the cone voltage produced $\mathrm{D}_{2 \beta-3}$. A scan rate of $1.5 \mathrm{~s} / \mathrm{scan}$ was used for both ES-MS and CID MS/MS experiments and the acquired spectra were summed for presentation.

For analysis, oligosaccharides were dissolved in ACN $/ \mathrm{H}_{2} \mathrm{O} 1: 1$, typically at a concentration of 5-10 $\mathrm{pmol} / \mu \mathrm{L}$, of which $5 \mu \mathrm{L}$ was loop-injected. Solvent (ACN/1 mM NH $\mathrm{mHCO}_{3}$ 1:1) was delivered by a Harvard syringe pump (Harvard Apparatus, Holliston, $\mathrm{MA})$ at a flow rate of $10 \mu \mathrm{L} / \mathrm{min}$.

\section{Results and Discussion}

A series of branched reducing oligosaccharides (Table 1) were analyzed to determine the structural information that could be derived from their ES-MS spectra. An optimal strategy to define structural features such as core-branching pattern, sequence, and linkage was established with this series that included nonfucosylated isomeric branched hexasaccharides $\mathrm{LNH}$ and $\mathrm{LNnH}$, and pairs of mono- and di-fucosylated analogues, MFLNH III/MFLNnH [a] and DFLNH [b]/DFLNnH, respectively. In addition, three oligosaccharides with the LNH sequence but containing two or three fucose residues at various positions were analyzed.

The nomenclature used to define fragmentation is based on that introduced by Domon and Costello [32]. In this instance, an $\alpha$ suffix is used to designate cleavages in the 6 -linked branch and a $\beta$ suffix for cleavages in the 3-linked branch of the Gal $\beta 1-4$ Glc core. Some fragments, derived from double cleavage such as ${ }^{0,2} \mathrm{~A}$ reported earlier [25], are accompanied by their dehydrated forms, $-\mathrm{h}$ being used to designate these ions in the CID mass spectra.

\section{Nonfucosylated Isomeric Hexasaccharides LNH and $\mathrm{LNnH}$}

LNH contains a type 1 chain on the 3-branch and a type 2 chain on the 6-branch, while $\mathrm{LNnH}$ has two type 2 branches (Table 1). The CID spectrum of [M- H] ${ }^{-} m / z$ 1071 of LNH has a set of C-type fragment ions (i.e., $\mathrm{C}_{1}$ : $m / z 179, C_{2}: m / z 382$, and $C_{3}: m / z$ 909, Figure 1a) giving evidence for the sequence. The branching pattern is indicated by the gap in mass between $\mathrm{C}_{2 \alpha}$ and $\mathrm{C}_{3}$ ions of $527 \mathrm{Da}$ corresponding to the trisaccharide composition $\mathrm{Gal}_{2} \cdot$ GlcNAc. In accord with results published in an earlier study, we also see that the ion pair resulting from ${ }^{0,2} \mathrm{~A}$-type fragmentation and its dehydrated product from the internal $-4 \mathrm{GlcNAc}$ - and terminal $-4 \mathrm{Glc}$ residues, ${ }^{0,2} \mathrm{~A}_{2 \alpha}$ at $m / z 281 / 263$ and ${ }^{0,2} \mathrm{~A}_{4}$ at $m / z 1011 /$ 993, respectively, are apparent. Other A-type fragment ions, such as an ${ }^{0,3} \mathrm{~A}_{3}$ ion at $m / z 454$ and ${ }^{2,4} \mathrm{~A}_{4}$ at $m / z 951$ from cross-ring cleavages are also useful for defining the linkage positions on the core Gal and Glc, respectively.

Clearly dominant in the negative-ion CID MS/MS spectrum of $[\mathrm{M}-\mathrm{H}]^{-}$of $\mathrm{LNH}$ is the fragment ion $\mathrm{D}_{2 \beta-3}$ at $m / z 526$ (Figure 1a). This characteristic fragment derives from a double glycosidic cleavage at the $C_{2 \beta}$ and $C_{3}$ positions as indicated and denoted as a $C-Z$ type cleavage ion [25]. Although the branching Gal residue is substituted at both the 3- and 6-positions, this double glycosidic cleavage occurs only at the 3- and not the 6-position (Figure 1a) and is similar to the previously observed D-type fragmentation for the 3-substituted GlcNAc- or Glc-containing sequences [25]. In addition, the ${ }^{0,3} \mathrm{~A}_{3}$ ion $(m / z 454)$ confirms the 6-branch composition. Cone voltage fragmentation (fragmentation induced in the cone region before the mass analyzer) also produces the major fragment ions observed in CID [25]. The $\mathrm{D}_{2 \beta-3}$ fragment $(m / z 526)$ produced by cone voltage fragmentation was used as precursor for CID MS/MS to give a product-ion spectrum in the quasi $\mathrm{MS}^{3}$ mode that unambiguously defines the sequence of the 6-linked chain (Figure $1 b$ ). The $C_{1 \alpha}$ and $C_{2 \alpha}$ cleavages indicate the Gal-GlcNAc sequence, and the ${ }^{0,2} \mathrm{~A}_{2 \alpha}$ doublet the -4 GlcNAc of a type 2 chain.

The product-ion spectrum (Figure $1 \mathrm{a}$ ) of $[\mathrm{M}-\mathrm{H}]^{-}$of $\mathrm{LNH}$ is dominated by fragment ions from the 6-linked branch, a feature of all the oligosaccharide spectra investigated (see below). Information on the 3-linked branch is missing, exemplified by the absence of the $\mathrm{D}_{1 \beta-2 \beta}$ ion $(\mathrm{m} / \mathrm{z} 202)$ that would be expected from a -3GlcNAc [25]. In contrast, the product-ion spectrum of the doubly charged molecular ion $[\mathrm{M}-2 \mathrm{H}]^{2-}(\mathrm{m} / \mathrm{z} 535)$ shows that fragments are produced from both branches, 

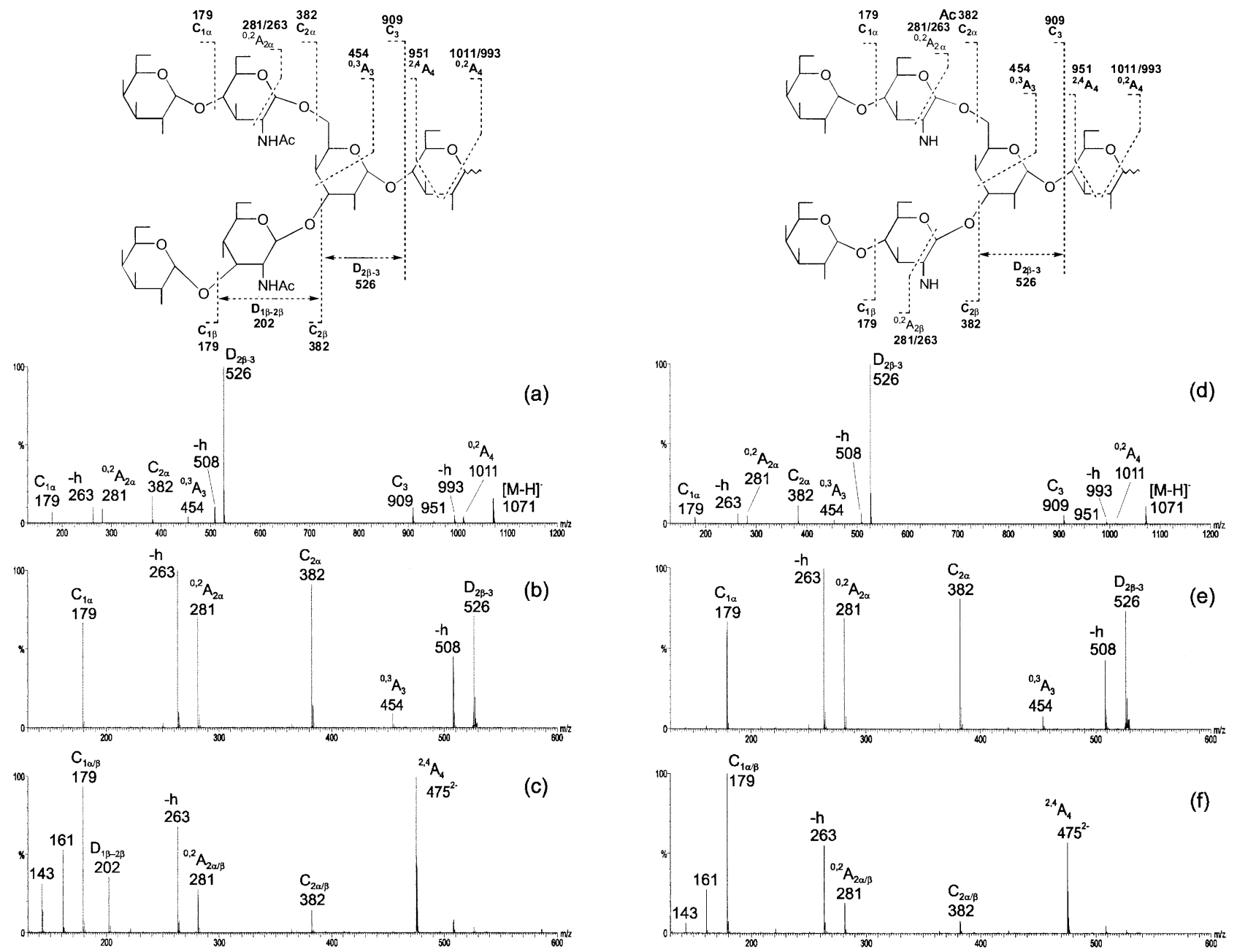

Figure 1. Electrospray CID MS/MS spectra of LNH and LNnH, (a): $[\mathrm{M}-\mathrm{H}]^{-}$; (b): $\mathrm{D}_{2 \beta-3}$ ion; (c): $[\mathrm{M}-2 \mathrm{H}]^{2-}$ of $\mathrm{LNH} ;(\mathbf{d}):[\mathrm{M}-\mathrm{H}]^{-} ;(\mathbf{e}): \mathrm{D}_{2 \beta-3}$ ion; $(\mathbf{f}):[\mathrm{M}-2 \mathrm{H}]^{2-}$ of LNnH. Structures are shown to indicate the proposed fragmentation.

not only the same ions as in the $[\mathrm{M}-\mathrm{H}]^{-}$spectrum but also $\mathrm{D}_{1 \beta-2 \beta}(\mathrm{m} / \mathrm{z} 202)$ from the -3GlcNAc- in the 3-linked branch (Figure 1c). In addition, a doubly charged ${ }^{2,4} \mathrm{~A}_{4}$ ion $(m / z 475)$ is intense when compared with its corresponding singly charged ion $\mathrm{m} / \mathrm{z} 951$ in the product-ion spectrum of $[\mathrm{M}-\mathrm{H}]^{-}$(Figure 1a).

Hence, the product-ion spectra of $[\mathrm{M}-\mathrm{H}]^{-}$and $[\mathrm{M}-2 \mathrm{H}]^{2-}$ of LNH give complementary information such that details of the 6-linked branch and the disaccharide core can be obtained from the $[\mathrm{M}-\mathrm{H}]^{-}$ spectrum (Figure 1a), and the sequence of the 3-linked branch can be derived from the additional fragmentation in the $[\mathrm{M}-2 \mathrm{H}]^{2-}$ spectrum (Figure 1c). Finally, the linkage and sequence of the 6-branch is unambiguously confirmed by CID MS/MS of the characteristic $D_{2 \beta-3}$ ion (Figure 1b).

For the isomeric hexasaccharide $\mathrm{LNnH}$, three production spectra were similarly acquired for $[\mathrm{M}-\mathrm{H}]^{-}$ (Figure 1d), $\mathrm{D}_{2 \beta-3}$ ion (Figure 1e), and $[\mathrm{M}-2 \mathrm{H}]^{2-}$ (Figure 1f) as precursors. As expected, the product ion spectrum of $[\mathrm{M}-\mathrm{H}]^{-}$is almost identical to that of $\mathrm{LNH}$ as fragmentation is dominated by the 6-branch which are the same in each oligosaccharide. Equally, as the $D_{2 \beta-3}$ fragment comprises the 6-linked branch and core branching sugar, product-ion spectra from LNH and $\mathrm{LNnH}$ are essentially identical. However, differences in the product-ion spectra of $[\mathrm{M}-2 \mathrm{H}]^{2-}$ ions distinguish their structures. In the spectrum of $\mathrm{LNH}, \mathrm{D}_{1 \beta-2 \beta}$ at $\mathrm{m} / \mathrm{z}$ 202 (Figure 1c) derives from the $-3 \mathrm{GlcNAc}$ in the 3-branch and the ion doublet ${ }^{0,2} \mathrm{~A}_{2 \alpha}$ at $m / z 281 / 263$ from the -4GlcNAc in the 6-branch, while in the spectrum of LNnH (Figure 1f) only the ${ }^{0,2} \mathrm{~A}$ ion doublet $(\mathrm{m} / \mathrm{z} 281$ / 263 ) is present, being produced from -4GlcNAc- of both 3 - and 6-branches.

\section{Monofucosylated Isomeric Heptasaccharides MFLNH III and MFLNnH [a]}

MFLNH III and MFLNnH [a] are analogs of LNH and LNnH (Table 1), respectively, being fucosylated at the 3-position of GlcNAc on the 6-branch. Their sequences 


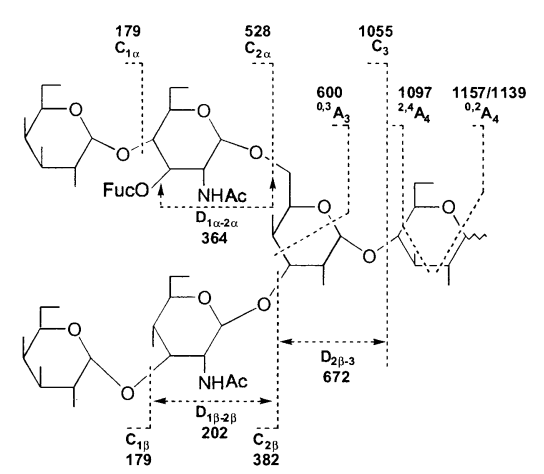

(a)
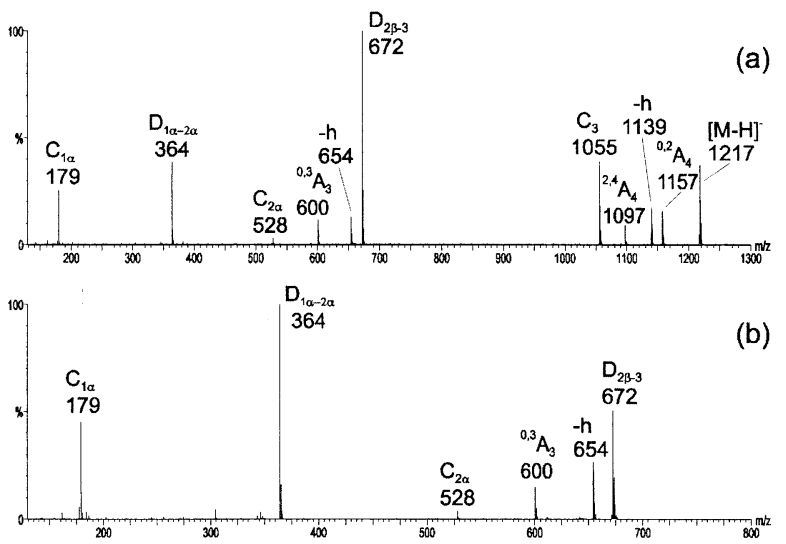

(b)

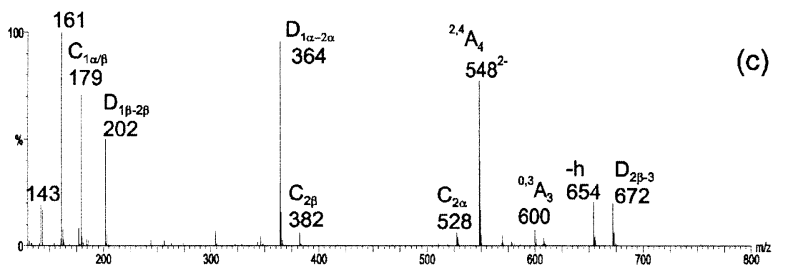

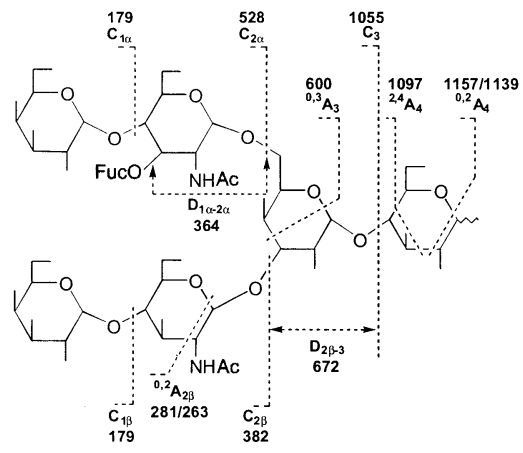
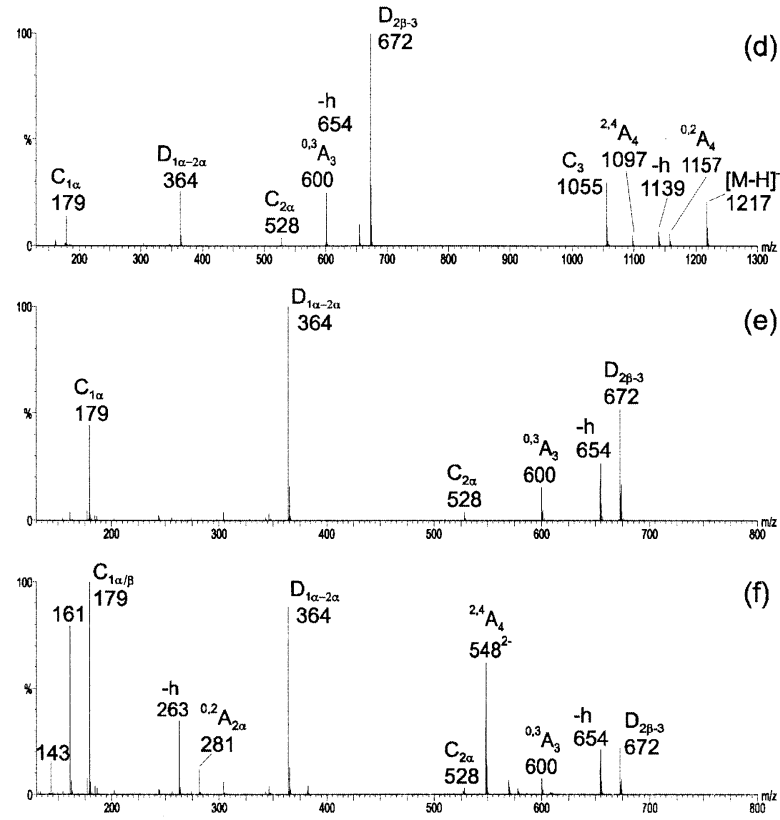

Figure 2. Electrospray CID MS/MS spectra of MLNH III and MFLNnH [a], (a): $[\mathrm{M}-\mathrm{H}]^{-} ;(\mathbf{b}): \mathrm{D}_{2 \beta-3}$ ion; (c): $[\mathrm{M}-2 \mathrm{H}]^{2-}$ of MFLNH III; (d): $[\mathrm{M}-\mathrm{H}]^{-} ;(\mathbf{e}): \mathrm{D}_{2 \beta-3}$ ion; $(\mathbf{f})$ : $[\mathrm{M}-2 \mathrm{H}]^{2-}$ of MFLNnH [a]. Structures are shown to indicate the proposed fragmentation.

and linkages can be deduced by the same approach. For example, the $\mathrm{Le}^{\mathrm{x}}$ blood-group determinant [i.e., Gal $\beta 1$ 4 (Fuc $\alpha 1-3)$ GlcNAc-] in the 6-branch of MFLNH III is reflected in the presence of the characteristic double cleavage $\mathrm{D}_{1 \alpha-2 \alpha}$ ion at $m / z 364$ [25] in the CID spectrum of $[\mathrm{M}-\mathrm{H}]^{-}$as precursor (Figure $2 \mathrm{a}$ ). The fucose on the 6-branch is also apparent from an intense $D_{2 \beta-3}$ ion at $m / z$ 672, an increase of $146 \mathrm{Da}$ from $m / z 526$ for a $\mathrm{D}_{2 \beta-3}$ ion of a Gal-GlcNAc- branch (cf Figure 1a and d), and from the same increase in mass of the ${ }^{0,3} \mathrm{~A}_{3}$ ion to $\mathrm{m} / \mathrm{z}$ 600. Product ions of $D_{2 \beta-3}$ from MFLNH III (Figure $2 b$ ) again confirm the structural features of the 6-branch with $\mathrm{C}_{1 \alpha}\left(m / z\right.$ 179) and $\mathrm{C}_{2 \alpha}(m / z 528)$ indicating a Fuc linked to the GlcNAc and $D_{1 \alpha-2 \alpha}(m / z 364)$ from the $\operatorname{Le}^{\mathrm{x}}$ configuration. Information on the 3 -branch is derived from the additional $D_{1 \beta-2 \beta}$ ion $m / z 202$, in the CID spectrum of $[\mathrm{M}-2 \mathrm{H}]^{2-}$ (Figure $2 \mathrm{c}$ ), as a type 1 chain with a mono-3-substituted GlcNAc.

Not surprisingly, the product-ion spectra of MFLNnH [a] from [M $-\mathrm{H}]^{-}$(Figure 1d) and $\mathrm{D}_{2 \beta-3}$ (Figure 1e) as precursors are similar to the respective spectra from MFLNH III due to their identical 6-linked chains. The difference in the 3-linked chains between the two heptasaccharides is only seen in the product-ion spectra of $[\mathrm{M}-2 \mathrm{H}]^{2-}$, with the -4 GlcNAc- of MFLNnH [a] giving a ${ }^{0,2} \mathrm{~A}_{2 \beta}$ ion doublet at $m / z 281 / 263$ (Figure $2 \mathrm{f}$ ) while the $-3 \mathrm{GlcNAc}$ - in MFLNH III produces a $\mathrm{D}_{1 \beta-2 \beta}$ ion at $m / z 202$ (Figure 2c).

\section{Difucosylated Isomeric Octasaccharides DFLNH [b] and DFLNnH}

DFLNH [b] and DFLNnH (Table 1) are difucosylated analogs of $\mathrm{LNH}$ and $\mathrm{LNnH}$, respectively. Each has one fucose in the 6-branch forming the $\mathrm{Le}^{\mathrm{x}}$ determinant while the other fucose on the 3-branch gives determinants $\mathrm{Le}^{\mathrm{a}}$ in DFLNH [b] and $\mathrm{Le}^{\mathrm{x}}$ in DFLNnH. As reported earlier [25], the diagnostic D-type fragment ions at $m / z 348$ and 364 again signal these Le $^{a}$ and Le ${ }^{x}$ trisaccharide sequences, respectively (see Figure 3).

The identical 6-branches and Gal1-4Glc core of 

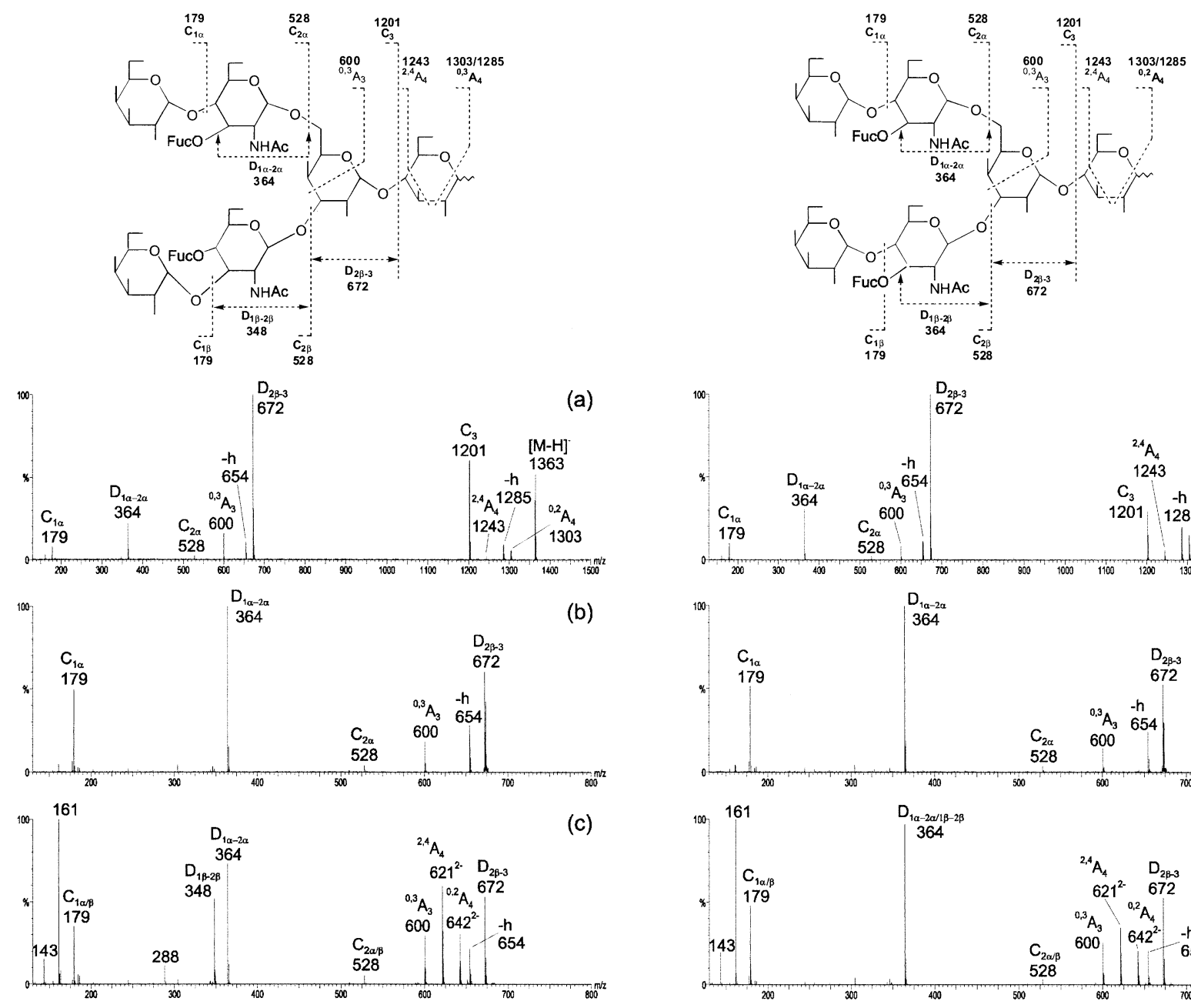

(a)
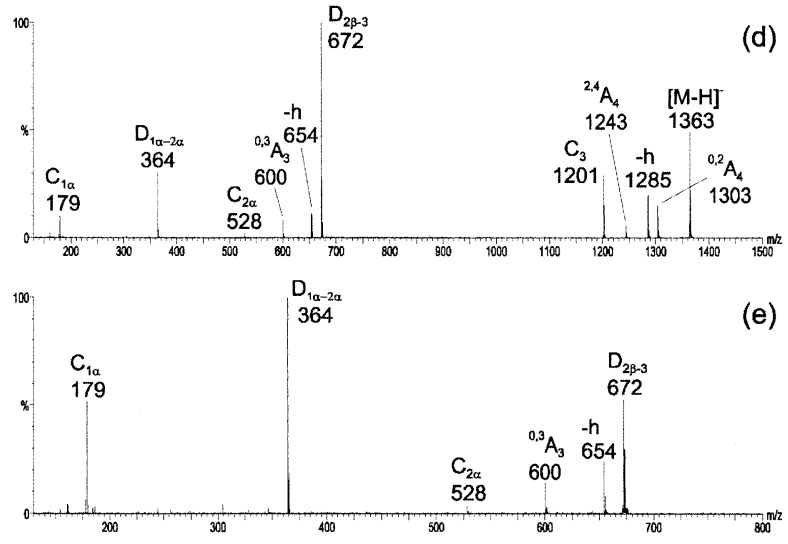

(c)

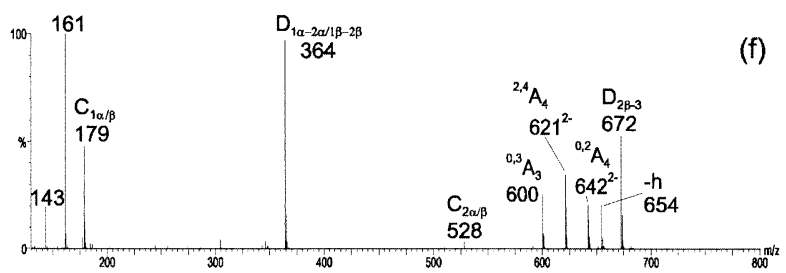

Figure 3. Electrospray CID MS/MS spectra of DFLNH [b] and DFLNnH, $(\mathbf{a}):[\mathrm{M}-\mathrm{H}]^{-} ;(\mathbf{b}): \mathrm{D}_{2 \beta-3}$ ion; (c): $[\mathrm{M}-2 \mathrm{H}]^{2-}$ of DFLNH [b]; (d): $[\mathrm{M}-\mathrm{H}]^{-} ;(\mathbf{e}): \mathrm{D}_{2 \beta-3}$ ion; $\left(\mathbf{f}:[\mathrm{M}-2 \mathrm{H}]^{2-}\right.$ of DFLNnH. Structures are shown to indicate the proposed fragmentation.

DFLNH [b] and DFLNnH lead to their very similar product-ion spectra of $[\mathrm{M}-\mathrm{H}]^{-}$(Figures $3 \mathrm{a}$ and $\mathrm{d}$, respectively) and $\mathrm{D}_{2 \beta-3}$ ions (Figures $3 b$ and e, respectively). $\mathrm{C}_{1 \alpha}, \mathrm{C}_{2 \alpha}$, and $\mathrm{C}_{3}$ ions indicate sequence with the fucosylated 6-branch being reflected in the masses of $\mathrm{C}_{2 \alpha}$ at $m / z 528, \mathrm{D}_{2 \beta-3}$ at $m / z 672$, and ${ }^{0,3} \mathrm{~A}_{3}$ at $m / z$ 600. The linkage position of the fucose at the 3-position of $-4 G l c N A c$ is readily deduced from the $\mathrm{D}_{1 \alpha-2 \alpha}$ ion at $m / z$ 364 as indicated above. The presence of the reducing terminal 4-substituted Glc is unambiguously identified by the unique ${ }^{0,2} \mathrm{~A}_{4}$ ion doublet $(\mathrm{m} / \mathrm{z} 1303 / 1285)$ together with a further ring cleavage fragment ion, ${ }^{2,4} \mathrm{~A}_{4}$, at $m / z 1243$.

The product-ion spectra of the $[\mathrm{M}-2 \mathrm{H}]^{2-}$ ions give information on the 3-branched chains of DFLNH [b] (Figure $3 c$ ) and DFLNnH (Figure 3f). The additional $\mathrm{D}_{1 \beta-2 \beta}$ ion at $m / z 348$ (Figure $3 \mathrm{c}$ ) indicates the fucose on the 3-branch chain is at the 4-position of -3GlcNAc (i.e., $\mathrm{Le}^{\mathrm{a}}$ configuration) of DFLNH [b]. As both branches in DFLNnH are type 2 chains containing the $\mathrm{Le}^{\mathrm{x}}$ sequence, the ion at $m / z 364$ in the MS/MS spectrum of [M -
$2 \mathrm{H}]^{2-}$ (Figure 3f) arises from both $\mathrm{D}_{1 \alpha-2 \alpha}$ and $\mathrm{D}_{1 \beta-2 \beta}$ fragmentation.

\section{Variously Fucosylated Hexasaccharides DFLNH [a], DFLNH [c], and TFLNH}

These three oligosaccharides are based on a branched LNH backbone, with type 2 and type 1 chains on the 6and 3-branches, respectively (Table 1). The combined application of CID MS/MS spectra of $[\mathrm{M}-\mathrm{H}]^{-},[\mathrm{M}-$ $2 \mathrm{H}]^{2-}$, and $\mathrm{D}_{2 \beta-3}$ ions is again able to define fully their structures. DFLNH [a] is fucosylated on the 6-branch giving a $\mathrm{Le}^{\mathrm{x}}$ sequence, and also on the 2-position of non-reducing terminal Gal of the 3-branch and hence displays the $\mathrm{H}$ determinant. The product-ion spectrum of $[\mathrm{M}-\mathrm{H}]^{-}$from DFLNH [a] (Figure 4a) shows the abundant $\mathrm{D}_{2 \beta-3}$ fragment $(m / z$ 672) indicating a Fuc in the 6-branch and $D_{1 \alpha-2 \alpha}(m / z 364)$ which identifies it to be at the 3-position of a -4GlcNAc-, assignments supported by the spectrum from $\mathrm{D}_{2 \beta-3}$ (Figure $4 \mathrm{~b}$ ). The 

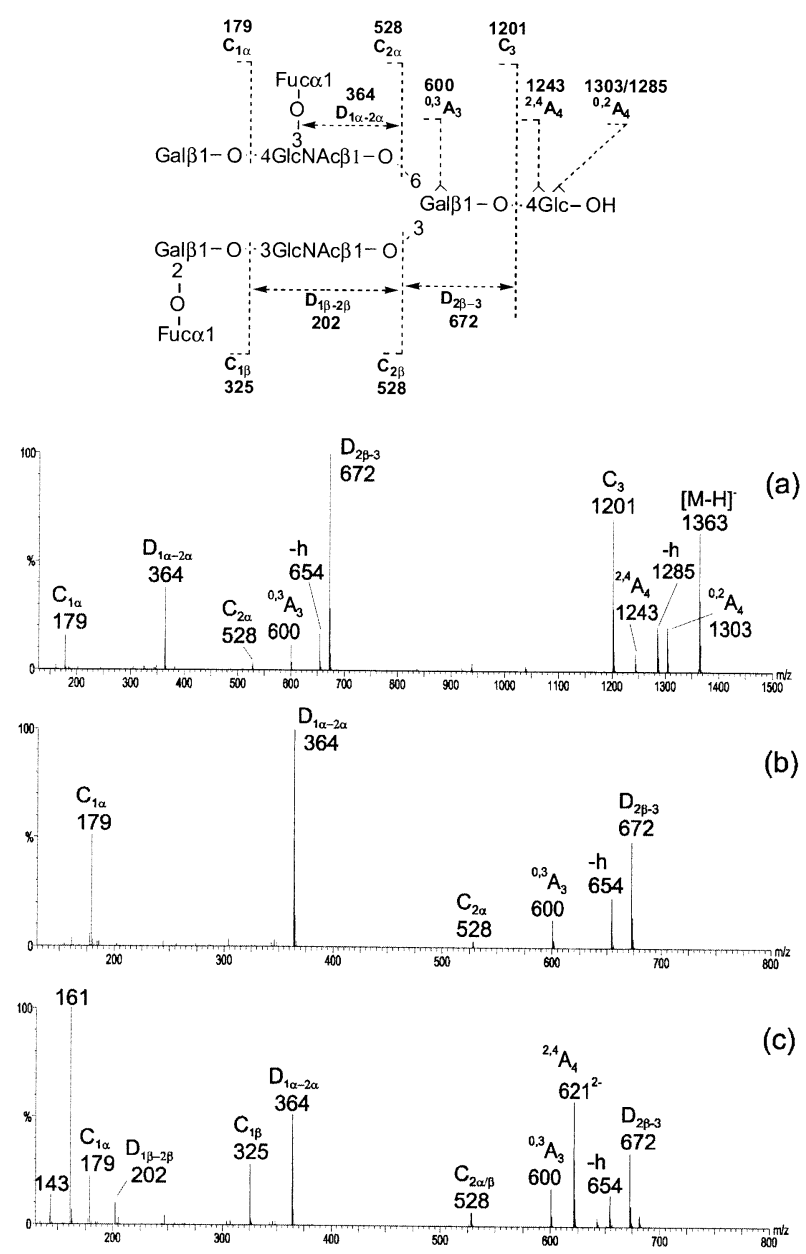

Figure 4. Electrospray CID MS/MS spectra of DFLNH [a], (a): $[\mathrm{M}-\mathrm{H}]^{-} ;(\mathbf{b}): \mathrm{D}_{2 \beta-3}$ ion; $(\mathbf{c}):[\mathrm{M}-2 \mathrm{H}]^{2-}$. The structure is shown to indicate the proposed fragmentation.

additional fragments, $\mathrm{m} / \mathrm{z} 202$ and $\mathrm{m} / \mathrm{z} 325$, in the spectrum of $[\mathrm{M}-2 \mathrm{H}]^{2-}$ (Figure $4 \mathrm{c}$ ) can be assigned as $\mathrm{D}_{1 \beta-2 \beta}$ and $\mathrm{C}_{1 \beta}$, respectively, indicating that the Fuc is linked to the terminal Gal and the internal GlcNAc is 3-substituted.

DFLNH [c] is also difucosylated (Table 1) but both fucoses are on the 3-branch as indicated by the dominant $\mathrm{D}_{2 \beta-3}$ fragment at $m / z 526$ (Figure $5 a$ ) that confirms the lack of a fucose in the 6-branch. In the spectrum of $[\mathrm{M}-\mathrm{H}]^{-}$(Figure $5 \mathrm{a}$ ), and also of $\mathrm{D}_{2 \beta-3}$ (Figure $5 \mathrm{~b}$ ), the unique ion doublet of $m / z 281 / 263\left({ }^{0,2} \mathrm{~A}_{2 \alpha}\right)$ identifies the -4GlcNAc- of the type 2 chain. In this case the additional fragment ions, $m / z 325\left(\mathrm{C}_{1 \beta}\right)$ and $m / z 348\left(\mathrm{D}_{1 \beta-2 \beta}\right)$, in the spectrum of $[\mathrm{M}-2 \mathrm{H}]^{2-}$ (Figure $5 \mathrm{c}$ ) define the locations of the two fucose residues in the 3-branch, with $\mathrm{m} / \mathrm{z} 325$ indicating the terminal Fuc-Gal- and $m / z 348$ the other fucose 4-linked to a -3GlcNAc-. This tetrasaccharide sequence on the 3-branch forms the $\mathrm{Le}^{\mathrm{b}}$ determinant.

TFLNH is a trifucosylated nonasaccharide with one fucose on the 6-branch and two on the 3-branch. The abundant $\mathrm{D}_{2 \beta-3}$ fragment at $m / z 672$ in combination with $\mathrm{C}_{3}$ at $m / z 1347$ seen in the product-ion spectrum of [M -
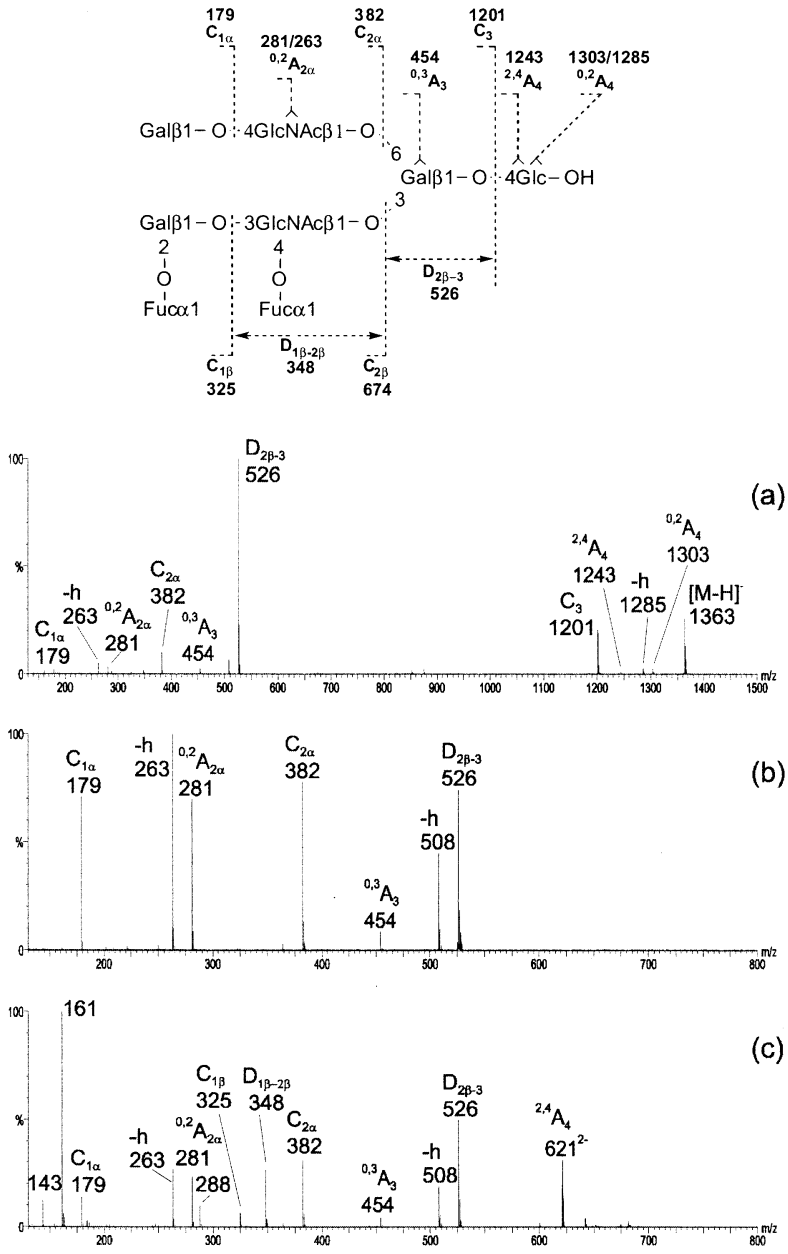

Figure 5. Electrospray CID MS/MS spectra of DFLNH [c], (a): $[\mathrm{M}-\mathrm{H}]^{-} ;(\mathbf{b}): \mathrm{D}_{2 \beta-3}$ ion; $(\mathbf{c}):[\mathrm{M}-2 \mathrm{H}]^{2-}$. The structure is shown to indicate the proposed fragmentation.

$\mathrm{H}]^{-}$(Figure 6a) permit the assignment of the fucose locations to 6- and 3-branched chains. The Fuc at the internal -GlcNAc- of the 6-branch is indicated by $\mathrm{C}_{1 \alpha}$ at $m / z 179$ and $C_{2 \alpha}$ at $m / z 528$, and its 3-linkage and hence Le $^{\mathrm{x}}$ sequence established by the $\mathrm{D}_{1 \alpha-2 \alpha}$ fragment at $\mathrm{m} / \mathrm{z}$ 364. CID MS/MS of $D_{2 \beta-3}, m / z 672$ (Figure 6b), is again confirmatory of the 6-linked chain. As before, sequence and linkage information of the 3-branch is obtained from the additional ions appearing in the $[\mathrm{M}-2 \mathrm{H}]^{2-}$ spectrum (Figure $6 \mathrm{c}$ ). $\mathrm{C}_{1 \beta}$ at $\mathrm{m} / \mathrm{z} 325$ reflects the Fuc linked to the terminal Gal while $\mathrm{D}_{1 \beta-2 \beta}$ at $m / z 348$ confirms the second fucose to be 3-linked to -4GlcNAc-. Thus, in TFLNH the $\mathrm{Le}^{\mathrm{x}}$ sequence on the 6-branch and $\mathrm{Le}^{\mathrm{b}}$ on the 3-branch are readily identified and distinguished.

\section{Conclusions}

Branching pattern, sequence, and partial linkage information is reflected in the negative-ion ES CID MS/MS of reducing oligosaccharides. The analysis can be achieved with high sensitivity (low pmol using normal 

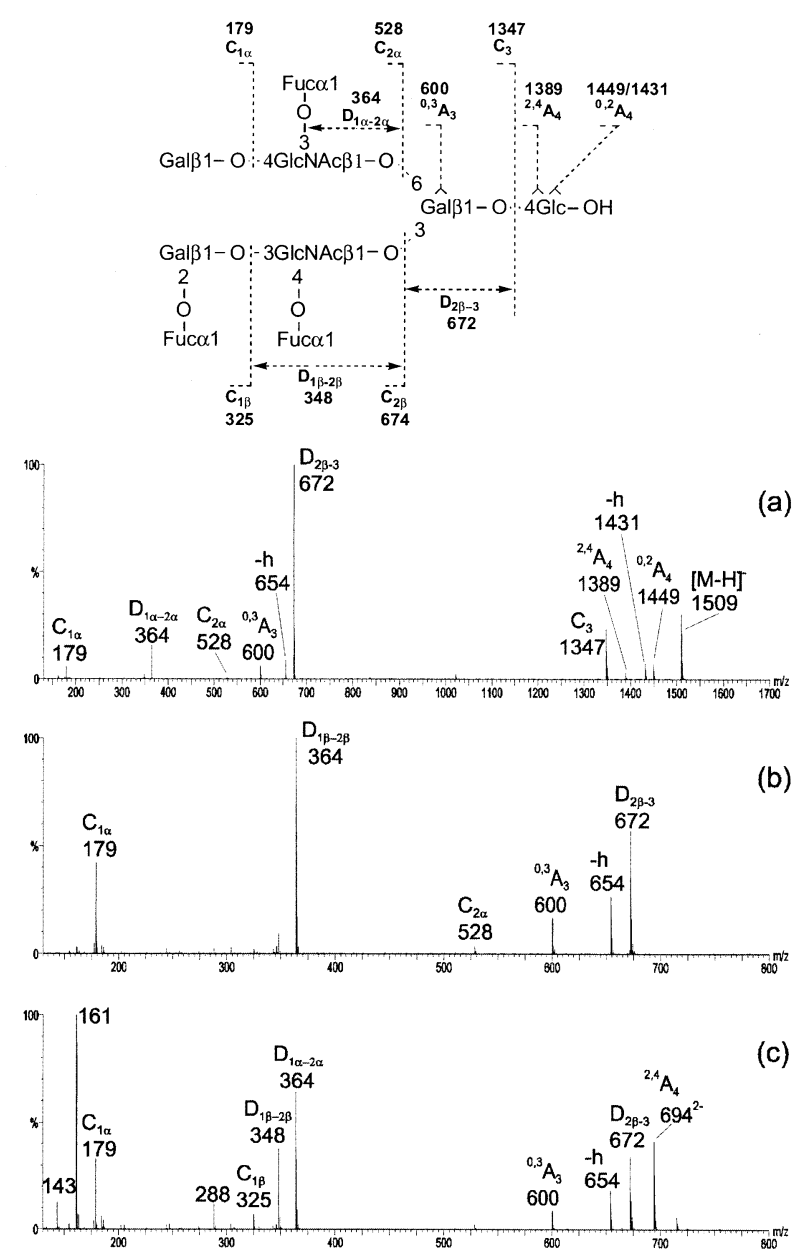

Figure 6. Electrospray CID MS/MS spectra of TFLNH, (a): [M $\mathrm{H}]^{-} ;(\mathbf{b}): \mathrm{D}_{2 \beta-3}$ ion; $(\mathbf{c}):[\mathrm{M}-2 \mathrm{H}]^{2-}$. The structure is shown to indicate the proposed fragmentation.

scale electrospray and $100 \mathrm{fmol}$ using nanospray) without derivatization on a quadrupole-orthogonal time-offlight instrument as previously demonstrated [25].

Although sialylated and sulfated oligosaccharides [33] are suited to negative-ion detection, the present work and our previous study [25] indicate that the weak acidity of neutral reducing oligosaccharides is also sufficient for formation of negatively charged ions under the MS conditions. This is in contrast to the earlier view that free oligosaccharides do not efficiently charge by either protonation or deprotonation [10]. For the oligosaccharides investigated, the reducing terminal Glc residue has a pKa of 12.28 and the weak acidity is largely derived from ionization of the anomeric hydroxyl group [34]; therefore, this reducing terminal hemiacetal hydroxyl is considered the preferred site of ionization. This is supported by absence of the same unique fragmentation pattern when the oligosaccharides are reduced (unpublished results). In the production spectra of the singly charged molecular ions of the reducing oligosaccharides investigated, the fragments are exclusively non-reducing terminal ions that do not contain the original ionization site. This may reflect the fact that the hemiacetal hydroxyl is a very weak acid, and a newly created charge at an oxygen site in a C-type or A-type fragment can compete for charge retention. As the cleavage occurs physically remote from the fixed charge site at one end of the molecular chain, it is proposed that the cleavage process is governed by charge-remote fragmentation [35]. However, in the case of $[\mathrm{M}-2 \mathrm{H}]^{2-}$, the second charge site is located at a different position or at one of several locations. Hence, the product-ion spectra of $[\mathrm{M}-2 \mathrm{H}]^{2-}$ are generated by two charge-remote sites or, more likely, by a combination of charge-remote and charge-driven reactions.

The important feature of the present study is the combined use of CID MS/MS of the singly and doubly charged molecular ions to deduce the branching pattern of 3,6-branched oligosaccharides. The uniqueness of the two types of CID spectra are the predominant presence of ions from the 6-linked branch and the absence of fragments from the 3-linked branch in the spectra of $[\mathrm{M}-\mathrm{H}]^{-}$, with fragmentation of both branches occurring in the spectra of $[\mathrm{M}-2 \mathrm{H}]^{2-}$. Also, double D-type cleavage at the 3-linked glycosidic bond of the branched Gal core residue produces an intense $D_{2 \beta-3}$ ion that is readily detectable, and with the molecular mass provides direct information on the branching pattern.

The ES CID MS/MS analysis of branched oligosaccharides can be summarized as follows:

(1) C-type fragmentation gives sequence information, and A-type cleavages give partial linkage information.

(2) Branching pattern can be derived from the characteristic $\mathrm{D}_{2 \beta-3}$ ion comprising the 6-linked chain and the core branching Gal residue in the product-ion spectrum of $[\mathrm{M}-\mathrm{H}]^{-}$. The monosaccharide composition of the 3-linked branch can be readily deduced from the mass difference between the sequence ions $\mathrm{C}_{2 \alpha}$ and $\mathrm{C}_{3}$. A saccharide ring fragment ion ${ }^{0,3} \mathrm{~A}_{3}$ is also useful for, and supportive of, the assignment of the 6-linked branch.

(3) Sequence and partial linkage information on the 6-branch is obtained directly from the product-ion spectrum of $[\mathrm{M}-\mathrm{H}]^{-}$with further confirmation by CID MS/MS of the $\mathrm{D}_{2 \beta-3}$ ion produced by cone voltage fragmentation.

(4) Structural information of the 3-linked branch is deduced from additional ions in the product-ion spectrum of $[\mathrm{M}-2 \mathrm{H}]^{2-}$.

(5) Structural details, such as the chain and bloodgroup types, are defined by the characteristic D- and $0,2 \mathrm{~A}$-type fragments in a similar fashion to the linear sugars reported previously [25].

\section{Acknowledgments}

This work was supported by a U.K. Medical Research Council program grant (G9601454). 


\section{References}

1. Gottschalk, A. The Influenza Virus Enzyme and Its Mucoprotein Substrate. Yale J. Biol. Med. 1954, 26, 352-364.

2. Watkins, W. M. Blood-Group Specific Substances. In Glycoproteins: Their composition, Structure and Function; Gottschalk, A., Ed.; Elsevier: Amsterdam, 1972; pp 830-899.

3. Kabat, E. A. Contributions of Quantitative Immunochemistry to Knowledge of Blood Group A, B, H, Le, I and i Antigens. Am. J. Clin. Pathol. 1982, 78, 281-292.

4. Feizi, T. Carbohydrate-Mediated Recognition Systems in Innate Immunity. Immunol. Rev. 2000, 173, 79-88.

5. Feizi, T. Demonstration by Monoclonal Antibodies that Carbohydrate Structures of Glycoproteins and Glycolipids are Onco-Developmental Antigens. Nature 1985, 314, 53-57.

6. DeFrees, S.; Kosch, W.; Way, W.; Paulson, J. C.; Sabesan, S.; Halcomb, R. L.; Huang, D. H.; Ichikawa, Y.; Wong, C. H. Ligand Recognition by E-Selectin-Synthesis, Inhibitory Activity, and Confirmational-Analysis of Bivalent Sialyl-Lewis- $X$ Analogs. J. Am. Chem. Soc. 1995, 117, 66-79.

7. Chai, W.; Feizi, T.; Yuen, C.-T.; Lawson, A. M. Nonreductive Release of O-Linked Oligosaccharides from Mucin Glycoproteins for Structural/Function Assignments as Neoglycolipids: Application in the Detection of Novel Ligands for E-Selectin. Glycobiol. 1997, 7, 861-872.

8. Dell, A.; Morris, H. R. Glycoprotein Structure Determination by Mass Spectrometry. Science 2001, 291, 2351-2356.

9. Duffin, K. L.; Welply, J. K.; Huang, E.; Henion, J. D. Characterization of N-Linked Oligosaccharides by Electrospray and Tandem Mass Spectrometry. Anal. Chem. 1992, 64, 1440-1448.

10. Reinhold, V. N.; Reinhold, B. B.; Costello, C. E. Carbohydrate Molecular Weight Profiling, Sequence, Linkage, and Branching Data: ES-MS and CID. Anal. Chem. 1995, 67, 1772-1784.

11. Weiskopf, A. S.; Vouros, P.; Harvey, D. J. Characterization of Oligosaccharide Composition and Structure by Quadrupole Ion Trap Mass Spectrometry. Rapid Commun. Mass Spectrom. 1997, 11, 1493-1504.

12. Viseux, N.; de Hoffmann, E.; Domon, B. Structural Analysis of Permethylated Oligosaccharides by Electrospray Tandem Mass Spectrometry. Anal. Chem. 1997, 69, 3139-3198.

13. Weiskopf, A. S.; Vouros, P.; Harvey, D. J. Electrospray Ionization-Ion Trap Mass Spectrometry for Structural Analysis of Complex N-linked Glycoprotein Oligosaccharides. Anal. Chem. 1998, 70, 4441-4447.

14. Yoshino, K.; Takao, T.; Murata, H.; Shimonshi, Y. Use of the Derivatizing Agent 4-Aminobenzoic Acid 2-(Diethylamino)ethyl Ester for High-Sensitivity Detection of Oligosaccharides by Electrospray Ionization Mass Spectrometry. Anal. Chem. 1995, 67, 4028-4031.

15. Ahn, Y. H.; Yoo, J. S. Malononitrile as a New Derivatizing Reagent for High-Sensitivity Analysis of Oligosaccharides by Electrospray Ionization Mass Spectrometry. Rapid Commun. Mass Spectrom. 1998, 12, 2011-2015.

16. Li, D. T.; Her, G. R. Structural Analysis of ChromophoreLabeled Disaccharides and Oligosaccharides by Electrospray Ionization Mass Spectrometry and High-Performance Liquid Chromatography/Electrospray Ionization Mass Spectrometry. J. Mass Spectrom. 1998, 33, 644-652.

17. Charlwood, J.; Langridge, J.; Tolson, D.; Birrell, H.; Camilleri, P. Profiling of 2-Aminoacridone Derivatized Glycans by Electrospray Ionization Mass Spectrometry. Rapid Commun. Mass Spectrom. 1999, 13, 107-112.

18. Saba, J. A.; Shen, X.; Jamieson, J. C.; Perreault, H. Effect of 1-Phenyl-3-Methyl-5-Pyrazolone Labeling on the Fragmentation Behavior of Asialo and Sialylated N-linked Glycans Under Electrospray Ionization Conditions. Rapid Commun. Mass Spectrom. 1999, 13, 704-711.
19. Shen, X.; Perreault, H. Electrospray Ionization Mass Spectrometry of 1-Phenyl-3-Methyl-5-Pyrazolone Derivatives of Neutral and N-Acetylated Oligosaccharides. J. Mass Spectrom. 1999, 34, 502-510.

20. Konig, S.; Leary, J. L. Evidence for Linkage Position Determination in Cobalt Coordination Pentasaccharides Using Ion Trap Mass Spectrometry. J. Am. Soc. Mass Spectrom. 1998, 9, 1125-1134.

21. Viseux, N.; de Hoffmann, E.; Domon, B. Structural Assignment of Permethylated Oligosaccharide Subunits Using Sequential Tandem Mass Spectrometry. Anal. Chem. 1998, 70, 4951-4959.

22. Mock, K. K.; Davey, M.; Cottrell, J. S. The Analysis of Underivatized Oligosaccharides by Matrix-Assisted Laser Desorption Mass Spectrometry. Biochem. Biophys. Res. Commun. 1991, 177, 644-651.

23. Harvey, D. J.; Küster, B.; Naven, T. J. P. Perspectives in the Glycosciences-Matrix-Assisted Laser Desorption/Ionization (MALDI) Mass Spectrometry of Carbohydrates. Glycoconj. J. 1998, 15, 333-338.

24. Tseng, K.; Hedrick, J. L.; Lebrilla, C. B. Catalog-Library Approach for the Rapid and Sensitive Structural Elucidation of Oligosaccahrides. Anal. Chem. 1999, 71, 3747-3754.

25. Chai, W.; Piskarev, V.; Lawson, A. M. Negative-Ion Electrospray Mass Spectrometry of Neutral Underivatized Oligosaccharides. Anal. Chem. 2001, 73, 651-657.

26. Bahr, U.; Pfenninger, A.; Karas, M.; Stahl, B. High-Sensitivity Analysis of Neutral Underivatized Oligosaccharides by Nanoelectrospray Mass Spectrometry. Anal. Chem. 1997, 69, 45304535 .

27. Lawson, A. M.; Chai, W.; Cashmore, G. C.; Stoll, M. S.; Hounsell, E. F.; Feizi, T. High-Sensitivity Structural Analysis of Oligosaccharide Probes (Neoglycolipids) by Liquid-Secondary-Ion Mass Spectrometry. Carbohydr. Res. 1990, 200, 47-57.

28. Chai, W.; Luo, J.; Lim, C. K.; Lawson, A. M. Characterization of Heparin Oligosachcaride Mixtures as Ammonium Salts Using Electrospray Mass Spectrometry. Anal. Chem. 1998, 70, 2060-2066.

29. Stoll, M. S.; Hounsell, E. F.; Lawson, A. M.; Chai, W.; Feizi, T. Microscale Sequencing of O-Linked Oligosaccharides Using Mild Periodate Oxidation of Alditols, Coupling to Phospholipid, and TLC-MS Analysis of the Resulting Neoglycolipids. Eur. J. Biochem. 1990, 189, 499-507.

30. Chai, W.; Stoll, M. S.; Cashmore, G. C.; Lawson, A. M. Specificity of Mild Periodate Oxidation of OligosaccharideAlditols: Relevance to the Analysis of the Core-Branching Pattern of O-Linked Glycoprotein Oligosaccharides. Carbohydr. Res. 1993, 239, 107-115.

31. Chai, W.; Yuen, C. T.; Feizi, T.; Lawson, A. M. Core-Branching Pattern and Sequence Analysis of Mannitol-Terminating Oligosaccharides by Neoglycolipid Technology. Anal. Biochem. 1999, 270, 314-322.

32. Domon, B.; Costello, C. E. A Systematic Nomenclature for Carbohydrate Fragmentation in FAB-MS/MS Spectra of Glycoconjugates. Glycoconj. J. 1988, 5, 397-409.

33. Thomsson, K. A.; Karlsson, H.; Hansson, G. C. Sequencing of Sulfated Oligosaccharides from Mucins by Liquid Chromatography and Electrospray Ionization Tandem Mass Spectrometry. Anal. Chem. 2000, 72, 4543-4549.

34. Hardy, M. R.; Townsend, R. R. High-pH Anion Exchange Chromatography of Glycoprotin-Derived Carbohydrates. Methods Enzymol. 1994, 230, 208-225.

35. Cheng, C.; Gross, M. L. Applications and Mechanisms of Charge-Remote Fragmentation. Mass Spectrom. Rev. 2000, 19, $398-420$. 